\title{
ANÁLISIS DE CONSERVACIÓN Y ESTRUCTURA CELULAR DE LA MADERA DE SEIS CANOAS MONÓXILAS DEL CENTRO SUR DE CHILE
}

\author{
NICOLÁS LIRA*, JUAN E. DIAZ-VAZ", SUSANA MUÑOZ****
}

\section{RESUMEN}

La madera, a pesar de ser un material orgánico biodegradable, es suficientemente resistente al deterioro bajo ciertas condiciones naturales. Restos y artefactos de madera saturados en agua y de origen cultural se han registrado en diferentes sitios arqueológicos del sur de Chile. Sin embargo, el estudio de este tipo de materiales culturales aún es insuficiente en nuestro país, ya que en la mayoría de los casos no se han aplicado metodologías adecuadas para su análisis y preservación. A partir de este estudio planteamos la necesidad de introducir nuevas perspectivas en torno a esta temática y esperamos iniciar una renovada etapa en las investigaciones arqueológicas de esta materialidad en el sur de Chile.

PALABRAS CLAVE: maderas chilenas, navegación indígena, sociedad mapuche prehispánica, wampos, bongos, centro-sur de Chile.

\section{CONSERVATION ANALYSIS AND CELULAR STRUCTURE OF WOOD FROM SIX LOGBOATS OF SOUTH CENTRAL CHILE}

\begin{abstract}
Although being an organic biodegradable material, wood can resist decay under certain natural conditions of the environment. Wood objects and waterlogged artifacts have been found in different archaeological sites in southern Chile. Thougth, the study of these type of materials in Chile is still restrained. In general the methodologies for its adequate study and preservation has not been applied. From this study we would like to propose the introduction of new approaches in this topic, and we hope to start a new level in the archaeological investigations in southern Chile.

KEY WORDS: chilean woods, indigenous navigation, mapuche prehispanic society, wampos, bongos, center-south of Chile.

Doctorando Université Paris 1 Panthéon-Sorbonne, UMR 8096 ARCHAM, 3 Rue Michelet 75006 Paris, France. nicoliras@ yahoo.com.

- Instituto de Tecnología de Productos Forestales, Facultad de Ciencias Forestales Universidad Austral de Chile, Casilla 567, Isla Teja, Valdivia, Chile. jdiazvaz@uach.cl.

... Dirección Museológica Universidad Austral de Chile, Casilla 586, Isla Teja, Valdivia, Chile. labconservacion@uach.cl.
\end{abstract}




\section{INTRODUCCIÓN}

La madera, a pesar de ser un material orgánico biodegradable, es suficientemente resistente al deterioro bajo ciertas condiciones naturales. Los sitios arqueológicos que presentan condiciones de gran humedad, o que se encuentran sumergidos o semisumergidos, las turberas, y en general las condiciones de saturación de agua permiten esta preservación en condiciones significativas. Así mismo las maderas que han sido expuestas al fuego y carbonizadas, o aquellas asociadas a productos de corrosión de metales permiten que se conserven de manera adecuada. Sin embargo, el análisis de este tipo de materiales culturales aún es insuficiente en Chile, ya que en la mayoría de los casos no se han aplicado metodologías adecuadas para su estudio y preservación. A partir de esta investigación planteamos la necesidad de introducir nuevas perspectivas en torno a esta temática y esperamos iniciar una nueva etapa en las investigaciones arqueológicas de esta materialidad en el sur de Chile. Proponemos la introducción de una serie de metodologías y técnicas para el estudio de maderas arqueológicas, ya bastante desarrolladas y conocidas en otros países, y que se agrupan bajo el concepto de arqueo-dendrometría, desarrollado por Lavier et al. (2004), que permite estudiar los restos de madera, no sólo como marcador cronológico y ecológico, sino también capaz de entregar información económica, cultural y tecnológica. La identificación de taxa, la tipología y morfología, la traceología de manufactura y uso, la dendrocronología, son todas herramientas analíticas que nos permiten aproximarnos al estudio de los artefactos de madera. De la misma forma la conservación de estos artefactos, manufacturados en materiales que se degradan fácilmente al ser extraídos de sus ambientes de depositación especiales, se convierte en un elemento esencial y prioritario para poder abordarlos de forma adecuada. En esta investigación intentamos dar una mirada integral, desde el contexto cultural, la anatomía de la madera y la conservación, al estudio de estos elementos a partir de las investigaciones realizadas sobre canoas monóxilas del centro-sur de Chile, embarcaciones de madera que se encontraban sumergidas o semisumergidas y que fueron extraídas sin un procedimiento controlado para su adecuada preservación.
En este estudio comenzamos por detallar el análisis de la taxa de la madera de canoas monóxilas ya mencionadas en publicaciones anteriores (Lira 2007, 2009, 2010a; Carabias et al. 2010) junto con su contexto cultural y una evaluación preliminar de las condiciones de conservación. Lamentablemente la extracción de restos de estas embarcaciones se ha realizado, en la mayoría de los casos, sin la asesoría de especialistas resultando en la pérdida de la información contextual, la ausencia de procedimientos de conservación adecuados y finalmente la falta de análisis y profundización en el estudio de estas piezas.

El término monóxila se refiere a una sola pieza de madera, es decir a canoas manufacturadas en un solo tronco ahuecado. Este tipo de canoas no eran las únicas embarcaciones utilizadas por los indígenas del sur de Chile a la llegada de los españoles. Los cronistas nos informan de una serie de balsas y naves que observaron tanto en las costas del litoral como en los ríos y lagos. Entre ellas podemos encontrar balsas de troncos, balsas de fibra vegetal, dalcas y canoas de corteza en el extremo sur. Sin embargo, las canoas monóxilas, wampos o bongos ${ }^{1}$ como también se les conoce en mapudungun son las que aparecen mejor documentadas y estudiadas. Las mejores descripciones de estas embarcaciones nos son entregadas por cronistas de la colonia.

Su manufactura y uso en el sur de Chile se encuentra bien documentado, desde la llegada de los españoles por los cronistas y sacerdotes de la época (Rosales, 1877-1878 [1678]), hasta algunas décadas atrás por la etnografía (Godoy, M. 2005 Ms, Bustamante et al. 2005). Se correspondería en tiempos históricos con el mundo mapuche-huilliche entre el Bio-Bio y el Seno de Reloncaví, quienes las habrían llamado wampos ${ }^{2}$, y se habrían utilizado tanto en los numerosos lagos cordilleranos y costeros como en los muchos ríos que cruzan de

1 El termino bongo [bóngo] corresponde, según la Real Academia de la Lengua Española, al término que designa una embarcación de fondo plano para el transporte fluvial, típica de algunos países americanos como Colombia y Venezuela.

Fray Felix José de Augusta en su Gramática Araucana traduce la palabra wampo como canoa (Augusta 1903: 136, 221 y 236). Wampu, según Lenz, es utilizado en mapundungun (lengua mapuche) para referirse a embarcación (canoa), así como para cualquier tronco de árbol ahuecado (Lenz 1897: 322). En esta investigación el término wampo se utilizará para referirse a las canoas monóxilas utilizadas como embarcación. 
cordillera a mar este territorio, así como también en la navegación costera. Los estudios sistemáticos y de carácter arqueológico de estas embarcaciones fueron iniciados a finales de la década de los 70 del siglo XX por Fernández a partir del hallazgo de la canoa de playa Bonita en el lago Nahuel Huapi, Argentina (Fernández 1978). Sin embargo, posteriormente estas investigaciones cayeron en un estancamiento, y no fueron retomados sino hasta el año 2004 a partir de la sistematización y evaluación de la información existente y dispersa en esta zona del Nahuel Huapi por Braikovich (2004). Posteriormente el hallazgo de dos canoas sumergidas y en contexto en el lago Calafquén (Chile) (Carabias et al. 2007) y un estudio de tipo regional, de sistematización y evaluación de la información para el centro sur de Chile (Lira 2007), le otorgan un nuevo impulso y consolidan este tipo de investigaciones (Lira 2009, Lira 2010 a y b) siendo objeto de una publicación en esta revista recientemente (Carabias et al. 2010) por lo que no nos adentraremos en los mismos aspectos generales.

En la actualidad las canoas monóxilas se han dejado de utilizar en Chile, y unas pocas se conservan en algunos museos del centro-sur. Sin embargo, aún perduran en la memoria de los habitantes más antiguos de estas zonas (Figuras 1 y 2).

\section{MATERIAL Y MÉTODO}

Se estableció un universo de seis piezas de canoas monóxilas que se conservan en diferentes

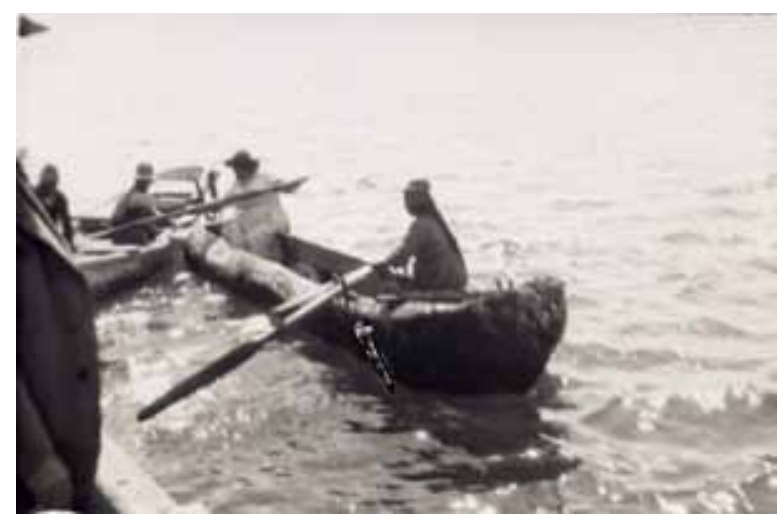

Fig. 1. Fotografía tomada por Rodolfo Knittel en Puerto Nuevo, Lago Ranco entre los años 19341936 y que correspondería a la segunda de una secuencia de tres fotografías. Colección de la Dirección

Museológica de la Universidad Austral de Chile. instituciones y museos del centro-sur de Chile, y que se encuentran en condiciones para ser estudiadas, abarcando una distribución geográfica desde Concepción por el norte hasta Maullín por el sur (Figura 3). Esta distribución territorial está supeditada a los lugares de conservación de las piezas, y se amplía hasta la región del Maule (en el caso de una canoa exhibida en el museo de Hualpén, Concepción) según las zonas de hallazgo, que se han verificado desde la década del sesenta del siglo XX hasta el 2005. Esta muestra puede parecer un tanto pequeña pero manejable y adecuada para realizar una primera aproximación al tema. La nomenclatura que se utilizó para la identificación de las piezas se refiere al lugar probable de uso o al lugar de hallazgo, Ej. Maullín_01 extraída de uno de los afluentes que abastece al río del mismo nombre (Lira 2007, 2010a). De esta manera las canoas analizadas en este estudio son: Maule_01 (Museo Parque Pedro del Rio Zañartu, Hualpén), Lleu-Lleu_01 (Museo Stom, Chiguayante), Lanalhue_01 (Museo Mapuche de Cañete), La Flor_01 (Museo Regional de la Araucanía, Temuco), Trancura_01 (Museo Leandro Panchulef, PUC Villarica), Maullín_01 (Museo Municipal de Maullín) (Lira 2007, 2010a) (Figura 3, Tabla 1). Su ubicación temporal sería entre los siglos XVI al XX D.C. de acuerdo a los fechados de $\mathrm{C} 14$ que se han realizado sobre las canoas La Flor_01, Lleu-Lleu_01 y Maullín_01 (Tabla 2). Aunque muy probablemente este tipo de embarcaciones habría estado en uso con anterioridad a estas fechas.

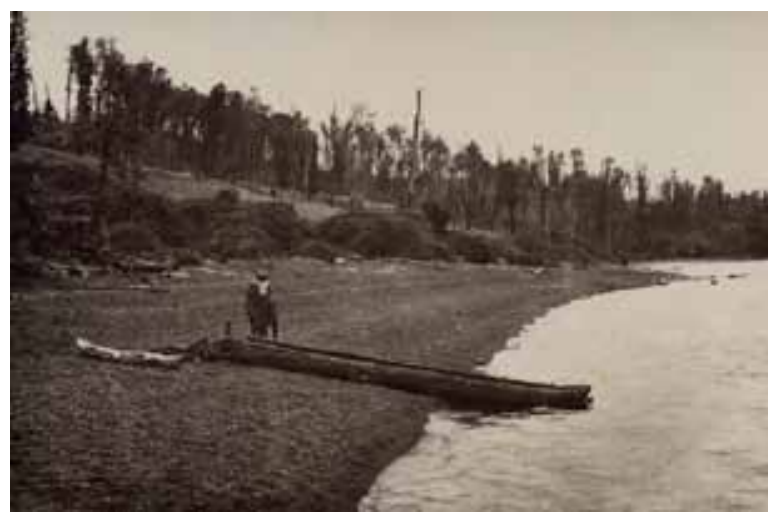

Fig. 2. Fotografía tomada por Rodolfo Knittel en Puerto Nuevo, Lago Ranco, el año 1934. Colección de la Dirección Museológica de la Universidad Austral de Chile. 


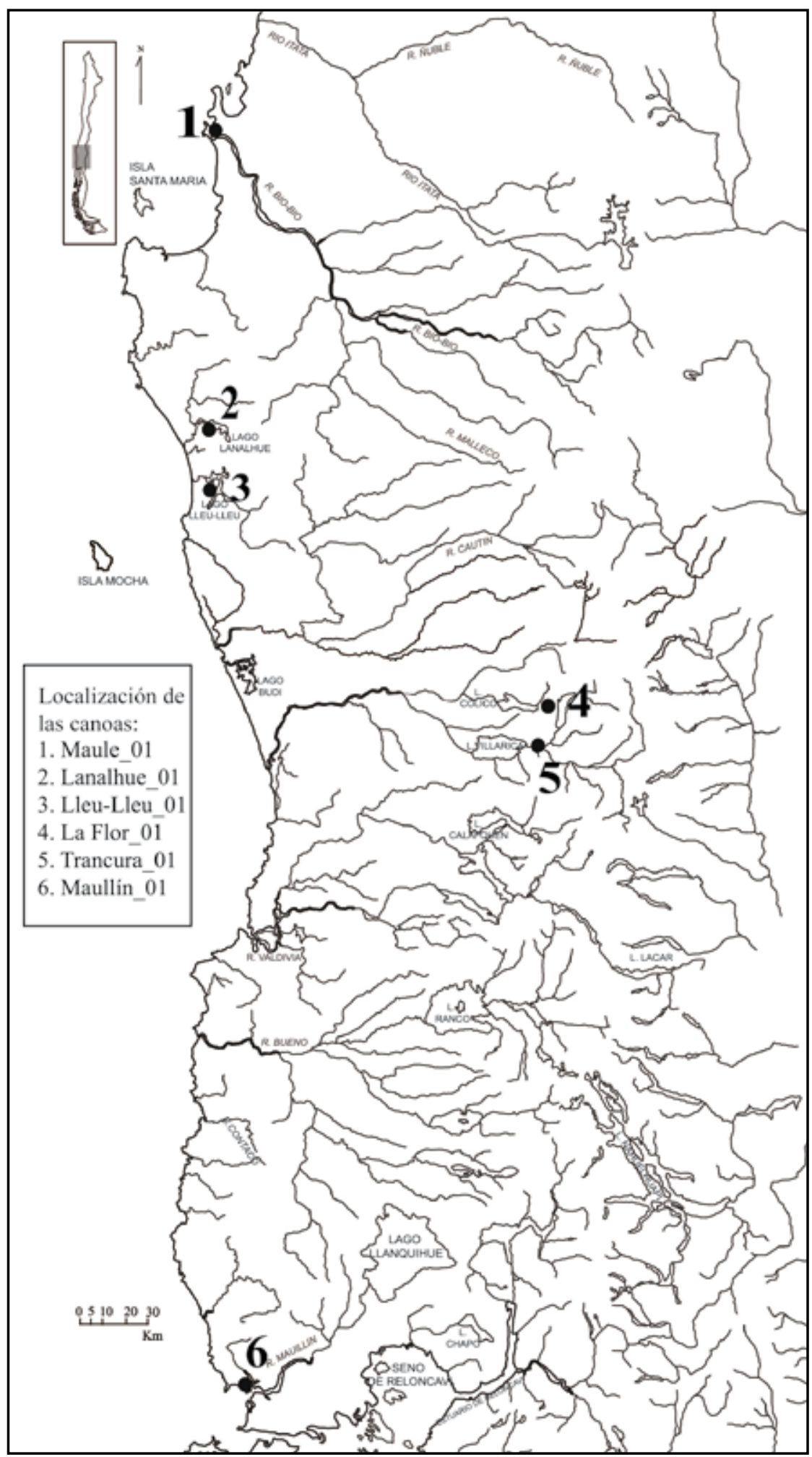

Fig. 3. Mapa de la zona de estudio con la localización de las canoas. 
Tabla 1. Características de las canoas en términos de número acorde a Figura 3, codificación y taxa.

\begin{tabular}{|c|c|c|}
\hline $\mathrm{N}^{\circ}$ & NOMBRE & TAXA \\
\hline 1 & Canoa Maule_01 & Nothofagus dombeyi (Mirb.) Oerts., coigüe \\
\hline 2 & Canoa Lanalhue_01 & Laurelia sempervirens (R. y P.) Tul, laurel \\
\hline 3 & Canoa Lleu Lleu_01 & Laurelia sempervirens (R. y P.) Tul, laurel \\
\hline 4 & Canoa La Flor_01 & Laurelia sempervirens (R. y P.) Tul, laurel \\
\hline 5 & Canoa Trancura_01 & Nothofagus nervosa (Phil.) Dim. y Mil.)., raulí \\
\hline 6 & Canoa Maullín_01 & Eucryphia cordifolia Cav., ulmo \\
\hline
\end{tabular}

Diagnóstico de conservación

El diagnóstico de conservación se realizó mediante la confección y aplicación de fichas especialmente diseñadas para este propósito en conjunto por los autores, teniendo en cuenta tanto el contexto de hallazgo de la pieza, su morfología, el transporte y lugar de depósito o exhibición actual. El llenado de estas fichas se realizó mediante el trabajo en terreno en los diferentes museos y la observación directa sobre las piezas.

\section{Preparación de muestras para microtomía}

La preparación de las muestras para su observación y estudio se inicio una vez que se identificó las embarcaciones, los detalles de sus morfologías y singularidades específicas. Se tomaron pequeñas muestras de las diversas piezas, destinadas a la observación microscópica, la identificación de las especies y el estado sanitario en que se encontraban.

Para cada una de las maderas se preparó, bajo estereomicroscopio, pequeños paralepípedos de unos $10 \mathrm{~mm}$ de sección. Estas muestras dimensionadas se infiltraron con polietilenglicol 4000 (PEG) manteniéndolas sumergidas en el PEG a una temperatura de $50^{\circ} \mathrm{C}$ durante algunas semanas. Con esta infiltración se logró aumentar la estabilidad de las maderas y permitir una condición de resistencia adecuada para su posterior corte microtómico. Los paralelepípedos infiltrados fueron destinados a estudiar el plano transversal (perpendicular al eje del tronco o rama), el plano radial (siguiendo la dirección del radio del tronco) y el plano tangencial (tangente a los anillos de crecimiento). De estos planos se obtuvo mediante microtomía láminas de entre 15 a $20 \mu \mathrm{m}$ de espesor las cuales permitieron la observación bajo microscopio.

Una vez cortadas, las láminas microtómicas se dejaron por unos minutos en una solución de $1 \mathrm{~g}$ de safranina en $50 \mathrm{ml}$ de agua destilada más $50 \mathrm{ml}$ de etanol. A continuación se realizó una deshidratación en una serie de soluciones alcohólicas hasta alcohol al $100 \%$ y finalmente se montaron en entellan. Estas preparaciones permanentes permitieron observar los distintos tipos de células presentes en cada una de las muestras de madera. Del mismo modo, se observó la estructura de las células, las modificaciones y posible presencia de microorganismos en los lúmenes y paredes de las células.

\section{Observación microscópica}

En la madera es posible reconocer una serie de distintos tipos de células. Entre las principales destacan: traqueidas, fibras, vasos, parénquima axial y radios leñosos. Las características particulares de cada una de ellas, así como su distribución, en la madera,

Tabla 2. Fechados radiocarbónicos realizados sobre tres de las canoas incluidas en este estudio.

\begin{tabular}{|c|c|c|c|c|}
\hline Embarcación & $\begin{array}{c}\text { Lab. } \\
\text { Beta N }\end{array}$ & $\begin{array}{c}\text { Fechado } \\
\text { Conv. AP }\end{array}$ & $\begin{array}{c}\text { Cal. 2 Sigmas } \\
\text { (95\% probabilidad) }\end{array}$ & $\begin{array}{c}\text { Intercepción de edad radiocarbónica } \\
\text { con curva de calibración }\end{array}$ \\
\hline $\begin{array}{c}\text { Lleu-Lleu_01 } \\
\text { (Lira 2007) }\end{array}$ & 220273 & $140+/-50$ & Cal. 1660 a 1950 DC & $\begin{array}{c}\text { Cal. 1680 DC; Cal. 1730 DC } \\
\text { Cal. 1810 DC;Cal. 1930 DC } \\
\text { Cal. 1950 DC }\end{array}$ \\
\hline $\begin{array}{c}\text { La Flor_01 } \\
\text { (Lira 2007) }\end{array}$ & 220272 & $270+/-50$ & $\begin{array}{c}\text { Cal. 1490 a 1680 DC } \\
\text { Cal. 1770 a 1800 DC } \\
\text { Cal. 1940 a 1950 DC }\end{array}$ & Cal. 1650 DC \\
\hline $\begin{array}{c}\text { Maullín_01 } \\
\text { (Lira 2007) }\end{array}$ & 220271 & $280+/-40$ & Cal. 1500 a 1670 DC & Cal. 1640 DC \\
\hline
\end{tabular}


forman parte de las singularidades que se emplean para reconocer y diferenciar unas maderas de otras.

La identificación de especies de madera se inicia, generalmente, constatando la presencia o ausencia de vasos, la distribución de ellos en el anillo de crecimiento, las diferencias que presentan en diámetro y tipo de asociación entre ellos. El tipo de punteaduras presentes en las paredes de los vasos puede permitir la identificación de la especie a que pertenece la madera considerando las diferencias que se presentan entre los tipos escalariforme, opuestas y alternas. La presencia o ausencia de estos tipos de punteaduras se utiliza entonces para caracterizar ciertas maderas. Del mismo modo, las paredes terminales de los vasos están perforadas distinguiéndose placas de perforación: escalariformes, simples y cribosas, que como en el caso anterior, la presencia o ausencia de alguna de ellas permite distinguir a ciertas madera de otras.

La presencia o ausencia de vasos separa a las maderas en dos grandes grupos. Por un lado la presencia de vasos indica que la madera observada pertenece a una especie latifoliada, y en el caso contrario, la ausencia de ellos indica que la madera corresponde a una conífera o que se trata de madera de canelo (Drimys winteri J.R. y G. Forster) la única especie latifoliada que no presenta vasos en el área de estudio.

El ordenamiento y cantidad de células parenquimáticas axiales presentes en el anillo de crecimiento revela, en muchos casos, el tipo de especie a que pertenece esa madera. Por ello su observación es relevante tanto para maderas latifoliadas como coníferas.

Los radios leñosos son también indispensables de utilizar en el reconocimiento de maderas. El número de células de ancho que pueden distinguirse en el plano transversal, permite separar, muchas de las veces, a coníferas de latifoliadas. El número de células de altura, apreciables en el plano longitudinal tangencial, son muchas de las veces típicas de ciertas especies. Diferentes células son reconocibles en los radios leñosos, entre ellas se tiene: radio traqueidas, procumbentes, erectas y cuadradas. La presencia de radio traqueidas distingue a especies coníferas exóticas de las especies coníferas nativas que no las poseen. Lo mismo sucede en el caso de la presencia de canales intercelulares no traumáticos, cuando los hay y en el caso de coníferas indican que la madera corresponde a una especie exótica (Diaz-Vaz et al.1991; 2001; Diaz-Vaz 2003).

\section{RESULTADOS}

\section{Resultados microscopía}

El análisis microscópico determinó, en todos los cortes transversales de todas las muestras observadas la presencia de vasos. Ello permite afirmar que todas ellas corresponden a maderas de especies latifoliadas.

La muestra obtenida del casco de la canoa de Maullín_01 se identificó como perteneciente a la especie ulmo (Eucryphia cordifolia Cav). Las principales características anatómicas que tipifican a esta madera, dicen relación a la presencia de vasos solitarios y también múltiples de 2 células. Estos muestran una sección angulosa. Las placas de perforación en esta muestra fueron en su mayoría escalariformes y simples lo que se corresponde con las características de la especie. Placas cribosas pueden encontrarse pero eran poco frecuentes en la muestra observada. Las punteaduras de los vasos se presentaron de tipo escalariformes, pero también opuestas y alternas, habitual en esta especie. Los radios leñosos se observaron heterogéneos, uniseriados y biseriados, con alturas de 10 células y los más altos de unas 20 células. El parénquima longitudinal se registro escaso y difuso.

La madera del casco de la canoa de Trancura_01 se identificó como perteneciente a la especie raulí (Nothofagus nervosa (P. y E.) Oerst) Las características anatómicas presentadas por esta madera coincidían con las reconocidas para la especie. Los vasos correspondieron a los del tipo múltiples de 2 a 6 células. Vasos del tipo solitarios fueron frecuentes y los agrupados se encontraron en menor proporción. Las placas de perforación de estos vasos eran simples y a veces escalariformes. Las punteaduras eran preferentemente alternas y opuestas. Las escalariformes se presentaban menos frecuentemente. Los radios leñosos eran uniseriados y heterogéneos. También se presentaron bi y triseriados. La altura de los radios leñosos fue de 17 células y de hasta 35 células como máximo.

En el caso de la canoa de La Flor_01 la identificación determinó que la especie utilizada correspondía a laurel (Laurelia sempervirens (R. y P.) Tul). Las características anatómicas observadas indicaron la presencia de vasos solitarios, de sección angulosa, también se encontraron vasos múltiples de 2 y 3 células. Las placas de perforación correspondieron 
a las del tipo escalariformes y las punteaduras tanto opuestas como escalariformes. Los radios leñosos se registraron como uniseriados a triseriados del tipo heterogéneo, con 10 a 20 células de altura. El parénquima longitudinal se presentó escaso y difuso.

La madera del casco de las canoas Lleu Lleu_01, y Lanalhue_01, se identificaron también como pertenecientes a la especie Laurel (Laurelia sempervirens (R. y P.) Tul) cuyas características anatómicas comparten con la madera de la canoa de La Flor_01.

La canoa de Maule_01 se identificó como madera perteneciente a la especie coigüe (Nothofagus dombeyi (Mirbel) Oerst) ya que presentó las típicas características que se reconocen en la especie. Sus vasos son solitarios múltiples en filas radiales de 2 a 6 células y agrupados menos frecuente. Los vasos presentan placas de perforación simples y punteaduras opuestas. Punteaduras del tipo escalariformes resultaron también frecuentes. Los radios leñosos eran

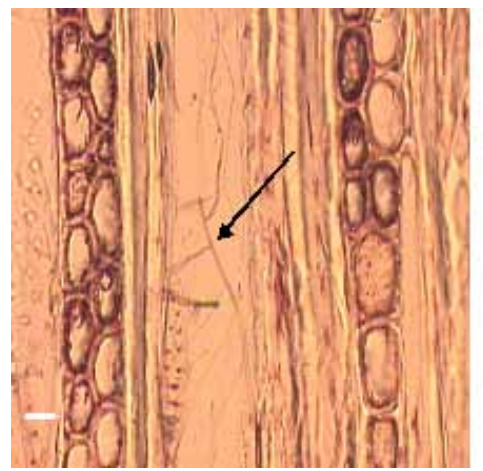

mm01 Maullín_01 ulmo (Eucryphia cordifolia Cav)

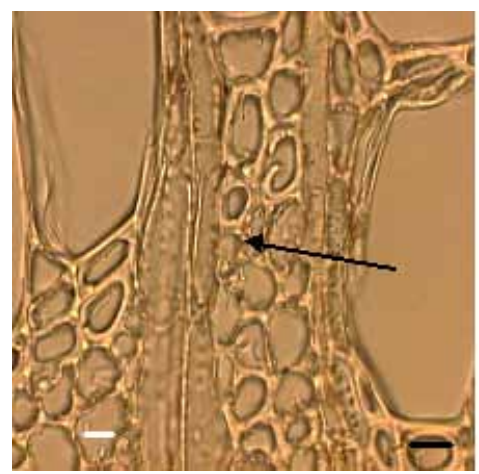

mm 04 Trancura_01 raul

(Nothofagus nervosa (Phil.) Dim. y Mil.) uniseriados a débilmente biseriados, heterogéneos con 3 a 20 células de altura. El parénquima longitudinal era escaso y difuso y por ello difícil de observar.

La generalidad de las muestras observadas, presentaron hifas de hongos en sus células en distintas cantidades según las muestras consideradas. Las hifas, secretan enzimas que transforman la madera en alimento útil para el hongo y así crecen colonizando de este modo la madera célula tras célula. De este modo se produce la pudrición de las paredes celulares y por ende de la madera, que pierde sus propiedades físicas y mecánicas entre otras (Figura 4).

La madera de la canoa de La Flor_01 identificada como madera de laurel presentó paredes celulares degradadas en algunas zonas, con degradaciones en forma de perforaciones y rupturas de grado medio. En todo caso la presencia de hifas no necesariamente coincidió con zonas que presentaron degradación (Figura 4).

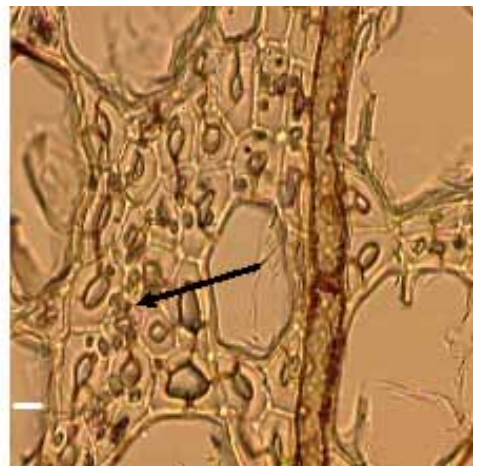

mm02 Maullín_01 ulmo (Eucryphia cordifolia Cav)

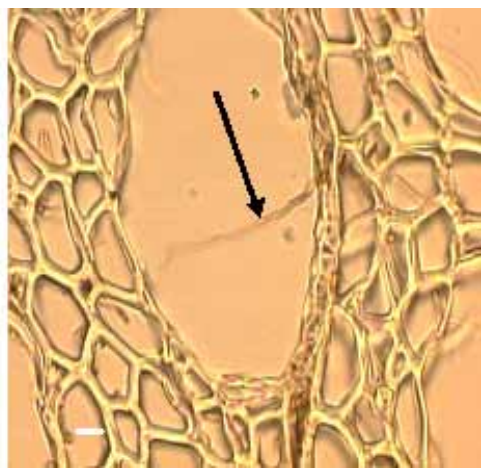

mm 06 Maule_01 coigüe

(Nothofagus dombeyi (Mirbel) Oerst)

Fig. 4. Presencia de hifas en células sin deterioro y células perforadas y degradadas por hongos de pudrición. 
La zona degradada de la madera de la canoa de La Flor_01, identificada como madera de laurel, presentó características similares a la madera de la canoa de Trancura_01 identificada como madera de raulí, y a la de la canoa de Maullín_01, ésta última identificada como ulmo.

En general las zonas de maderas con degradación se presentaron en células no parenquimáticas. También fue consistente la presencia de hifas dentro de células que no tenía degradación de sus paredes. Del mismo modo, células con deterioro producido por hongos no necesariamente presentaron hifas en sus lúmenes o en células vecinas (Diaz-Vaz 2000; 2003; Diaz-Vaz et al. 2001).

\section{Análisis de Conservación}

Cada una de las canoas estudiadas presentaron características asociadas ya sea por el conocimiento y manejo de la especie y su comportamiento, así como de las particularidades de cada una de ellas; ambos aspectos pudieron haber sido tomados en cuenta al momento de elegir el árbol para construir la embarcación. Es así como por ejemplo en las piezas Trancura _01 y Maule_01 elaboradas de raulí (Nothofagus nervosa ( $P$. et E.) Oerst.) y coigüe (Nothofagus dombeyi (Mirb.) Oerts.), respectivamente, se registró evidencia de que fueron usadas, sino totalmente al menos parcialmente, con su corteza original, es decir se conservó esta protección natural en las áreas donde por factura no fue necesario eliminarla. Probablemente se fue desprendiendo de a poco durante su utilización. Esta exfoliación puso en evidencia en uno de los casos (Trancura 01) la acción de insectos xilófagos que actuaron sobre el individuo vivo, entre la corteza y el duramen, que dejaron huellas que posteriormente se fueron ablacionando (Figura 5). En cambio las Canoas Colico_01, Lleu Lleu_01 y Lanalhue_01 elaboradas en laurel (Laurelia sempervirens (R. y P.) Tul), carecen completamente de esta protección natural. Esto puede explicarse por las características de la corteza de esta especie, la que es relativamente blanda y en la medida que el individuo se desarrolla se va desprendiendo en capas. A pesar de ser una especie que presenta una mayor resistencia a ser colonizada por hongos e insectos, al igual que por ejemplo en el caso del ulmo (Eucryphia cordifolia Cav.) de Maullín_01, el ataque de Ganoderma australe produce un proceso de degradación, esto es la destrucción del tejido y la delignificación y separación de fibras en las áreas que rodean a la zona afectada. Este es uno de los hongos causante de pudrición blanca.

En cuanto a los contextos de hallazgo, extracción y métodos de conservación, la relación es variable y sólo hay coincidencia en que los hallazgos han sido fortuitos. En la mayoría de los casos las canoas fueron extraídas sin un procedimiento metodológico de investigación y de conservación (Lira 2007, 2009, 2010a).

En tres de ellas: Maullín_01, Lleu_lleu_01 y Trancura_01 hay pruebas certeras que su extracción fue con el propósito de comercialización y sólo la canoa Lanalhue_01 ha tenido un tratamiento guiado por profesionales, con un procedimiento aún en fase

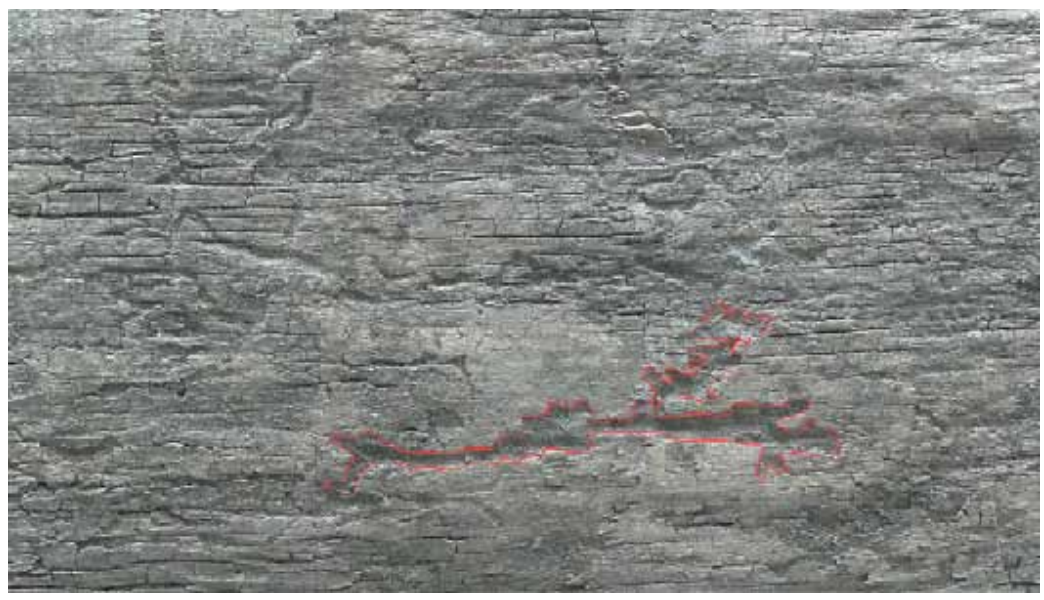

Fig. 5. Canales de acción de los coleópteros barrenadores, canoa Trancura_01. 
inicial, ya que no se realizó el estudio del contexto cultural y se destruyó la posibilidad de fechar la pieza a raíz del tratamiento de conservación de la madera (Bahamóndez y Valenzuela 2001).

El estado de preservación de las canoas es también muy variable. Como se ha dado cuenta hasta ahora, depende fundamentalmente de las características del material lignocelulósico, de las condiciones en que fue abandonada o perdida y la forma de depositación. En el caso de la canoa Trancura_01, esta se encontró volteada y sepultada (Lira 2007) en la ribera del Trancura, río que desagua en el lago Villarrica, y que constituye una zona de gran sedimentación e hidrodinámica. Al ser extraída de su lugar de depositación, la canoa pasó en poco tiempo desde un medio húmedo relativamente anaeróxico y estable a un medio ambiente variable en relación a temperatura, humedad relativa y a la luz. Esto produjo una evaporación del agua contenida en las paredes celulares de la madera y la contracción de las fibras lo que afectó fuertemente las capas externas comprimiéndose su volumen en sentido paralelo y presentando fracturas longitudinales. Por esto presenta cierta deformación de su eje que se contradice con el cuidado de selección del tronco y factura de este tipo de pieza, por lo que se estima que corresponde a una deformación por asentamiento en el medio subacuático que debió aumentar en el proceso de secado de la pieza.

Las maderas que componen las canoas en estudio, presentan por su estado de conservación, en general cambios en su color natural y también una reducción de sus resistencias mecánicas. La exposición de maderas antiguas a la radiación ultravioleta provoca un cambio de la coloración, adquiriendo una coloración superficial grisácea producto de la degradación de la lignina. La coloración café oscura opaca es habitual en maderas que se encuentran a la intemperie y con alto contenido de humedad. En la superficie aparecen en forma recurrente numerosas grietas angostas y de poca profundidad debido al secado por la exposición al sol y la baja humedad ambiental.

Todas las piezas presentan evidencias de suciedad superficial y suciedad adherida, la presencia de sales, fotodegradación de la superficie y algún tipo de pudrición (parda o cúbica y blanca). La pudrición de la madera puede originarse por efecto de distintos organismos, entre ellos los hongos, que se distinguen en aquellos que atacan directa y primeramente los compuestos celulósicos. La madera queda con la lignina sin degradación y por ello la madera atacada adquiere una tonalidad parda. Otros hongos atacan primeramente la lignina y por ello la madera queda con una coloración blanca. Todos los tipos de pudriciones producen el deterioro de la madera afectando sus propiedades mecánicas y físicas.

Las canoas La Flor_01 y Maullín_01 registran la presencia de líquenes y musgos. Todas las canoas presentan arañas en su superficie, las que muy rápido y fácilmente colonizan los objetos de madera, mientras que solamente la madera de la canoa Maule_01 ha sido atacada por coleópteros. También se aprecian problemas en los soportes de exhibición de las piezas, que en la mayoría de los casos no son adecuados y que podrían estar acentuando algunos procesos de deformación. Esta situación ha sido mejorada en los casos de las canoas Lanalhue_01 y La Flor_01, a partir de la renovación de los museos respectivos y su exhibición, y Maullín_01 gracias a un proyecto Fondart para su puesta en valor ejecutado por los autores (Lira y Muñoz 2008). Se plantea la necesidad de emplear en las exhibiciones museográficas soportes adecuados, que distribuyan el peso de la pieza en forma equilibrada y se adapte a la forma de cada pieza.

Tabla 3. Propiedades de las especies usadas para la factura de canoas en el centro-sur de Chile (Diaz-Vaz et al. 2002).

\begin{tabular}{|c|c|c|c|c|c|c|c|}
\hline Taxa & $\begin{array}{c}\text { Densidad } \\
\mathrm{Kg} . / \mathrm{m}^{3}\end{array}$ & Humedad \% & $\begin{array}{c}\text { Contracción } \\
\text { volumétrica \% }\end{array}$ & Dureza Kg. & $\begin{array}{c}\text { Flección } \\
\text { Etática } \mathrm{N} / \mathrm{cm}^{2}\end{array}$ & $\begin{array}{c}\text { Compresión } \\
\mathrm{N} / \mathrm{cm}^{2}\end{array}$ & Cizalle $\mathrm{N} / \mathrm{cm}^{3}$ \\
\hline $\begin{array}{l}\text { Raulí Nothofagus nervosa } \\
\text { (Phil.) Dim. y Mil.) }\end{array}$ & 550 & 12 & 12 & 400 & 80 & 45 & 10 \\
\hline $\begin{array}{l}\text { Ulmo Eucryphia } \\
\text { cordifolia Cav }\end{array}$ & 720 & 12 & 17 & 350 & 80 & 55 & 13 \\
\hline $\begin{array}{c}\text { Laurel Laurelia } \\
\text { sempervirens (R. y P.) Tul }\end{array}$ & 580 & 12 & 11 & 240 & 70 & 40 & 10 \\
\hline $\begin{array}{c}\text { Coigüe Nothofagus } \\
\text { dombeyi (Mirb.) Oerts }\end{array}$ & 660 & 12 & 15 & 500 & 80 & 55 & 12 \\
\hline
\end{tabular}




\section{DISCUSIÓN Y CONCLUSIONES}

La identificación de las especies de árboles a que pertenecen las maderas utilizadas en la fabricación de las canoas en estudio es un paso relevante en la reconstrucción de la historia de los seres humanos que las diseñaron, construyeron y utilizaron. De esta manera, la identificación de la especie a que pertenece un objeto de madera permite relacionar estos vestigios arqueológicos con situaciones de interés para la descripción de eventos pasados. Esto ha permitido contrastar los registros históricos acerca de la construcción de embarcaciones con ciertas maderas específicas, además de poder asociarla a alguna región donde estas especies se encuentren en abundancia. Por otra parte ha posibilitado la profundización en los aspectos del conocimiento y manejo de la madera y sus propiedades, y del bosque en general por parte de las poblaciones que utilizaron estas embarcaciones.

De la misma forma los elementos anatómicos que conforman la madera, aportan información relevante tanto para la identificación de la especie vegetal a que pertenece, así como también permiten indagar sobre las condiciones en que se han desarrollado y mantenido las maderas de estos artefactos (Diaz-Vaz 1979, 2003).

A partir de los resultados obtenidos del análisis de identificación de especie para cada una de las muestras de madera de las canoas, se confirma la estrecha relación existente entre las comunidades humanas que las utilizaron y los recursos del bosque, ya adelantados por Aldunate (1989, 1996), Villagrán (1991 y 1998) Aldunate y Villagrán (1992), Rappaport y Ladio (1999), y que ha sido elaborada en una propuesta arqueológica por Adán et al. (2004, 2010) en lo que se ha denominado la Tradición de Bosque Templado. Podríamos plantear que las poblaciones que están manufacturando este tipo de embarcaciones muestran cierto grado de especialización en el uso de los recursos madereros, ya que están seleccionando especies con características particulares. Si bien la muestra estudiada es pequeña y aún insuficiente para establecer resultados concluyentes, sí se pueden observar tendencias que nos permiten esbozar ciertas hipótesis. Al respecto se puede plantear que se estaría privilegiando maderas que presentan propiedades que facilitan su tallado y manufactura (como una menor densidad y dureza), así como con una resistencia mecánica mediana y que no presenten mayores complejidades en su secado como el laurel (Laurelia sempervirens Looser), ulmo (Eucryphia cordifolia Cav.), y raulí (Nothofagus nervosa (P. y E.) Oerst.) (Tablas 1 y 3). Las características de durabilidad natural, esto es resistencia al ataque de hongos $e$ insectos que nos parecían tan importantes a priori pasarían a un segundo plano ante la predilección por las propiedades ya mencionadas. Esto podría estar atentando contra la conservación de estas embarcaciones, lo que resultaría en que sólo encontremos canoas de tiempos recientes ya que la madera no soportaría las condiciones de inmersión o semi inmersión en las que se encuentran depositadas por largos períodos (Lira 2007, 2009, 2010a). Sin embargo, la madera a pesar de ser un material orgánico biodegradable, es suficientemente resistente al deterioro bajo ciertas condiciones ambientales naturales especiales para su conservación como ya se explicó en la introducción.

Junto con esto, otros factores que habrían influido en la selección de las especie arbóreas para la elaboración de embarcaciones probablemente consideraron, además de la facilidad para trabajar la madera, la disponibilidad de las diversas especies en las distintas áreas, así como el peso del tronco y su transporte al lugar donde sería utilizado, muchas veces a través de terrenos poco accesibles. El árbol, sería seleccionado por las características alcanzadas en su estado adulto y que podían ser asociadas a los requerimientos de la embarcación. Otros aspectos interesantes son aquellos que tendrían que ver con el significado simbólico y el carácter sagrado que las comunidades indígenas le han atribuido a algunos de éstos árboles, cómo es el caso del laurel (Laurelia sempervirens Looser) (Hall y Witte 1998) utilizado en ceremonias como el nguillatún, y que podría ser otro elemento importante para su selección.

Para continuar con los avances en la especificidad del tema y de sus contenidos se ha creado la necesidad de elaborar un protocolo interdisciplinario que contribuya a la normalización de las estrategias de investigación de las canoas monóxilas. Es así como ya se han revisado aspectos básicos como volumen, forma y zona de extracción de las muestras que permita la preservación constructiva y estética de la pieza.

Del análisis de algunas de las estructuras más deterioradas se ha establecido la necesidad de realizar mapas de variabilidad de humedad, con el propósito 
de establecer un parámetro de comparación del tipoestructura y reacción-medio, y registrar por medio de un método comprobable la necesidad de tratamiento o adecuación del medio ambiente para su conservación.

Un aspecto concluyente de esta investigación es la necesidad de crear y aplicar políticas y criterios de actuación ya usadas y normalizadas internacionalmente, privilegiando la conservación in situ de los restos arqueológicos sumergidos como lo sugiere el anexo a la convención de la UNESCO sobre la Protección del Patrimonio Cultural Subacuático (2001), en la medida que la estructura no esté en riesgo de deterioro y se evalúe periódicamente su estabilidad con el medio biológico. Si no es posible la conservación in situ, la experiencia de la investigación extraída del estudio de las canoas monóxilas "en tierra" ha permitido establecer que el estudio de las características de la costa o ribera, profundidad en la que se encuentra, tipo de agua, especie maderable, estado en que se encuentra la estructura y anatomía celular de la madera, junto con su fechado, análisis de elementos incorporados, deterioros estructurales, análisis de sedimento, -todos análisis in situ-, son determinantes para llevar a cabo un buen procedimiento de extracción y prever el comportamiento estructural de la pieza desde el medio líquido, pudiendo prever además, sobre la base del estudio de los niveles de contracción de cada tipo de madera y el tiempo de inmersión, su comportamiento. De la misma forma, estudios específicos sobre la madera, como la traceología de manufactura y uso, morfología de la madera, y en algunos casos la dendrocronología, deben realizarse cuando la madera aún se encuentra saturada en agua, ya que luego de su secado se pierde gran parte de esta información por los cambios que sufre la materia.

Por otro lado, es importante notar que la mayoría de los cambios, deterioros y procesos de degradación de estas piezas se han producido durante su extracción y luego en los lugares de exhibición y depósito. Especial atención se debe tener con los depósitos y salas de exhibición, que, a través de pequeños detalles y cuidados que no requieren de grandes gastos en recursos, pueden mejorar en gran medida la conservación de estas piezas. De la misma forma el uso de soportes adecuados que no sigan aumentando el deterioro de las piezas y que las protejan del público que las visita es esencial.

Por último es importante recordar que en esta investigación intentamos dar una mirada integral, desde el contexto cultural, la anatomía de la madera y la conservación, al estudio de estos elementos a partir de las investigaciones realizadas sobre canoas monóxilas del centro- sur de Chile. Nos parece fundamental continuar aplicando enfoques de este tipo que permitan aproximarse e interpretar desde una mirada multidisciplinaria estos artefactos, y a través de ellos a quienes los construyeron y utilizaron, para su estudio en el presente y su preservación para el futuro.

\section{AGRADECIMIENTOS}

Esta investigación se desarrolló en el marco del proyecto de investigación FONDECYT 1060216 del gobierno de Chile. Los autores quieren agradecer a cada uno de los museos que visitamos para estudiar y registrar estas canoas. También queremos agradecer a Leonor Adán, directora del proyecto por su apoyo y revisiones, Valentina Figueroa por su ayuda en las imágenes, figuras y revisiones, y a Fernanda Kalazich por su revisión.

\section{BIBLIOGRAFÍA}

ADÁN, L., C. GARCÍA Y R. MERA. 2010. La Tradición Arqueológica de Bosques Templados y su estudio en la región lacustre cordillerana de las regiones IX y XIV (centro-sur de Chile). En Actas del XVII Congreso Nacional de Arqueología Chilena, Tomo 2, pp.14611471. Ediciones Kultrún, Valdivia.

ADÁN, L., R. MERA, M. BECERRA Y M. GODOY. 2004. Ocupación Arcaica en territorios boscosos y lacustres de la región precordillerana andina del centro-sur de Chile: El sitio Marifilo 1 de la localidad de Pucura. Actas XV Congreso de Arqueología Chilena, Chungara 36(T2):1121-1136.

ALDUNATE, C. 1989. Estadio alfarero en el sur de Chile. En Culturas de Chile. Prehistoria. J. Hidalgo, V. Schiapacasse, H. Niemeyer, C. Aldunate y I. Solimano (eds.), Pp 329-348. Editorial Andrés Bello, Santiago.

1996. Mapuche: gente de la tierra. En Culturas de Chile. Etnografía. J. Hidalgo, V. Schiappacasse, H. Niemeyer, C. Aldunate y P. Mege editores. Pp 111-134. Editorial Andrés Bello, Santiago.

ALDUNATE, C. Y C. VILLAGRÁN. 1992. Recolectores de los Bosques Templados del Cono Sur Americano. En Botánica Indígena de Chile de Ernesto Wilhelm de Moesbach. C. Aldunate and C. Villagrán (eds.). Pp 2338. Editorial Andrés Bello, Santiago. 
AUGUSTA, FRAY F.J. DE. 1903. Gramática Araucana. Imprenta Central, J.Lampert, Valdivia.

BAHAMÓNDEZ, M. y P. VALENZUELA. 2001 Conservación del Patrimonio sumergido: un nuevo desafío en Chile. En: Actas de las Primeras Jornadas Latinoamericanas de Arqueología Subacuática. pp. 109-115. Ediciones de la Universidad Internacional SEK, Santiago.

BRAICOVICH, R. 2006. Las Canos Monóxilas del lago Nahuel Huapi. En Arqueología Histórica en América latina Temas y Diluciones recientes. P Furnari y F. Brittez editores. Pp 49-70. Ediciones Suárez, Mar del Plata.

BUSTAMANTE A., F. DE LA MAZA, G. GONZALEZ, L. LUNA, G. SILVA Y T. VEINTIE. 2005. La Comunidad Mapuche de Putue cuenta su Historia. Equipo de trabajo de la Pontificia Universidad Católica de Chile, Comité de Desarrollo Agrícola de Putue Comunidad Indígena Pedro Ancalef, Putue, Villarrica.

CARABIAS, D; N. LIRA Y L. ADÁN. 2010. Reflexiones en torno al uso de embarcaciones monóxilas en ambientes boscosos lacustres precordilleranos andinos, zona centro sur de Chile. Magallania 38 (1): 87-108.

CARABIAS, D., M. CHAPANOFF Y L. ADAN. 2007. Evidencias de navegación en ambientes lacustres precordilleranos andinos: evaluación arqueológica subacuática del sitio "Dos canoas del lago Calafquén". En: Levantando piedras, desenterrando huesos... y develando arcanos, F. Morello, M. Martinic, A. Prieto y G. Bahamonde editores. Pp 503-514. Ediciones CEQUA, Punta Arenas.

DIAZ-VAZ, J.E. 1979. Claves de Identificación de Maderas de Árboles Nativos y Cultivados en Chile. Bosque 3(1):15-25.

DIAZ-VAZ J.E., SCHMITT,U., RUETZE,M., DILLEHAY,T., BREMER,J., FAIX,O. 1991 Microscopy and Analytical Pyrolisis of Palaeowood from Nothofagus dombeyi and Amomyrtus luma. Holzforschung 45(6):407-414.

DIAZ-VAZ, J.E. 2000. Anatomía de madera inoculada con hongos. Seminario Biopulpaje Kraft Aplicado a Pinus radiata. Empresas Arauco. Arauco.

DIAZ-VAZ, J.E., J. GONZALEZ, S. ESPINOSA, y A. FERNANDEZ. 2001. Biopulps for Pinus radiata: Anatomical aspect, 2 Deutsch-Chilenisches Symposium Ecologically and Economically Sustainable Forestry Ytopia or feasible option, Goettingen, Alemania. Simposium Universidad de Goettingen - Universidad Austral de Chile. Goettingen.

DIAZ-VAZ, J.E., H. POBLETE, R. JUACIDA Y F. DEVLIEGER. 2002. Maderas Comerciales de Chile. Chilean Comercial Woods. Marisa Cuneo Ediciones, Valdivia.

DIAZ-VAZ, J.E. 2003. Anatomía de maderas. Universidad Austral de Chile, Editorial Marisa Cuneo, Valdivia.
FERNÁNDEZ, J. 1978. Restos de embarcaciones primitivas en el Lago Nahuel Huapi. Anales de Parques Nacionales XIV:45-77.

GODOY, M. 2005. Informe Etnográfico Proyecto FONDECYT Nº10402326 año 1. Cuenca del Río Valdivia, Sector Cordillera, comuna de Panguipulli. MS.

HALL, M. Y J. WITTE. 1998. Maderas del Sur de Chile: árboles, aplicaciones y procesos. Editorial Universitaria, Santiago.

LAVIER, C., C. LOCATELLI Y D. POUSSET. 2004. De l'artefact en bois à la nature forestière: quelques histoires parlantes. En: La Revue Forestière Française, LVI, numéro spécial, "le bois dans son futur". Pp.17-29. ENGREF, Nancy.

LENZ, R. 1897. Estudios Araucanos Materiales para el Estudio de la Lengua, la Literatura i las Costumbres de los indios. En: Anales de la Universidad de Chile, Tomo XCVII. Imprenta Cervantes, Santiago.

LIRA, N. 2007. Canoas monóxilas en el centro sur de Chile: Navegando sobre los árboles. Memoria para optar al titulo de Arqueólogo, profesora guía Victoria Castro, Universidad de Chile, Santiago. MS

2009. Embarcations de tradition indigène dans la $\mathrm{Pa}$ tagonie du nord : connexions, contacts et routes entre le versant oriental et occidental des Andes. Mémoire de Master 2 Préhistoire- Ethnologie-Anthropologie, Université de Paris 1 Panthéon- Sorbonne, Paris. MS. 2010a. Canoas Monóxilas en el centro-sur de Chile: Navegando sobre los árboles. Actas del XVII Congreso Nacional de Arqueología Chilena, Tomo 2: 1473-1485. Ediciones Kultrún, Valdivia.

2010b. Ríos, Lagos, Bosques y Volcanes: Paisaje Cultural en La Araucanía. Actas del $6^{\circ}$ Congreso Chileno de Antropología, Tomo II: 1184-1194, Valdivia.

LIRA, N Y S. MUÑOZ. Una canoa Indígena en el Río Maullín, rescate de una historia. Informe Final Fondo Nacional de la Cultura y las Artes, Línea Conservación y Difusión del Patrimonio Cultural. MS.

RAPAPORT, E. Y A. LADIO. 1999. Los bosques andino-patagónicos como fuentes de alimento. Bosque 20(2): 55-64.

ROSALES, D. 1877 - 1878 [1678]. Historia general del reyno de Chile, Flandes Indiano. Tomos I y II. Publicada anotada y precedida por B. Vicuña Mackenna. Imprenta de El Mercurio, Valparaíso.

VILLAGRÁN, C. 1991. Historia de los bosques templados del sur de Chile durante el Tardiglacial y Postglacial. Revista Chilena de Historia Natural 64:447-460.

1998. Etnobotánica indígena de los bosques de Chile: sistema de clasificación de un recurso de uso múltiple. Revista Chilena de Historia Natural 71: 245-268. 